\title{
Research Article \\ Synthesis of Planar Reflective Gratings for Silicon Interconnects
}

\author{
Ronald Millett, Jamie Ramsey, Przemek Bock, Julie Nkanta, Henry Schriemer, \\ Trevor J. Hall, and Serge Bidnyk
}

School of Information Technology and Engineering, University of Ottawa, Ottawa, Canada ON K1N 6N5

Correspondence should be addressed to Ronald Millett, rmillett@site.uottawa.ca

Received 29 November 2007; Accepted 13 March 2008

Recommended by Pavel Cheben

The design and simulations of planar reflective gratings for building optical interconnects in silicon-on-insulator (SOI) were studied for a range of silicon core thicknesses of 0.1 to $10 \mu \mathrm{m}$. The verticality of the grating facets has been shown to be the main contributing factor to the cumulative crosstalk in thick silicon cores. The dispersion property of the slab was found to limit the minimal thickness of the core for polarization-insensitive gratings. The effects of polarization-dependent confinement on optical crosstalk were studied. The findings were used to design and simulate a polarization-insensitive 18-channel coarse wavelength division demultiplexer (CWDM) with a free spectral range of over $600 \mathrm{~nm}$. The CWDM demultiplexer uses a $1.7 \mu \mathrm{m}$ silicon core and combines a shallow-etch tapered rib structure and multimode silicon channels to produce box-like passbands for integrated receiver applications. The diffraction grating was constructed using double astigmatic point design with phase-corrected grating facets to reduce astigmatism. Optical properties of the planar gratings have been simulated using quasivectorial diffraction grating theory. The simulation results confirm that there is high diffraction efficiency and low optical crosstalk over the entire range of operation. Applications of planar silicon gratings to the synthesis of silicon interconnects are discussed.

Copyright (c) 2008 Ronald Millett et al. This is an open access article distributed under the Creative Commons Attribution License, which permits unrestricted use, distribution, and reproduction in any medium, provided the original work is properly cited.

\section{INTRODUCTION}

Over the past several decades, data transfer rates over electronic interconnects have been increasing exponentially following Moore's law. The clock speed of silicon-based electronic processors has reached microwave frequencies of over $4 \mathrm{GHz}$ causing severe problems with the signal integrity of electronic interconnects. At these frequencies, traditional stripline interconnects suffer from a fundamental crosscoupling penalty and large radiation losses. The research in silicon photonics has been driven primarily by the objective of overcoming the limitations of stripline interconnects. Recently, several key elements, including silicon lasers, modulators, and photodetectors, have been realized in silicon using low-cost CMOS-compatible processes [1, 2]. Further research in silicon-on-insulator (SOI) shows promise for implementing additional key functionalitydemultiplexing - to enable wavelength division multiplexing and transferring data over multiple channels. The high index contrast of SOI waveguides also permits smaller device footprints compared to more weakly guided material systems.

Due to performance limitations, arrayed waveguide grating- (AWG-) based demultiplexers in SOI have not been able to compete effectively with silica-based AWGs. Porting AWG technology directly from silica to SOI has been a challenge, mainly due to high waveguide propagation losses, limited spectral range, strong polarization dependence, and poor optical crosstalk arising from phase noise in the delay line region of SOI-based AWGs [3]. In order to overcome AWG limitations in SOI, several research groups have explored the possibility of using planar reflective gratings as an alternative to AWGs. To generate diffraction, planar reflective gratings, also known as Echelle gratings, rely on the optical properties of a 2D silicon slab as opposed to a phase array of waveguides in AWGs. SOI-based planar reflective gratings have now been realized with SOI core thicknesses of $0.22 \mu \mathrm{m}$ [4], $3 \mu \mathrm{m}$ [5], and $5 \mu \mathrm{m}$ [6]. These initial studies are very promising and demonstrate that on-chip insertion 
losses of less than $2 \mathrm{~dB}$ are readily achievable in both thin and thick core SOI-based planar gratings $[4,5]$.

In this paper, we theoretically explore the optical properties of planar reflective grating demultiplexers based on silicon cores of various thicknesses. We show that the thickness of the silicon core layer has a profound effect on the polarization and crosstalk performance of the SOI grating. The results of our findings were used to synthesize a polarization-insensitive 18-channel coarse wavelength division demultiplexer (CWDM) with box-like responses based on a planar reflective grating. The device combines a shallow-etch tapered rib structure and multimode silicon channels. The grating was optimized to reduce aberration effects. The polarization-insensitive performance of the demultiplexer was confirmed using quasi-vectorial diffraction theory. Applications of planar reflective gratings to building silicon interconnects are discussed.

\section{PLANAR REFLECTIVE GRATING DESIGN}

The success of AWGs in meeting the requirements of the multichannel demultiplexer market has been largely due to the silica platform where AWGs can be fabricated using shallow etched waveguides in a relatively simple fabrication process. In spite of a considerably smaller footprint, the fabrication of planar reflective gratings in silica was more complex compared to AWGs and required additional research into controlling the verticality and smoothness of the reflective facets. Contrary to silica, there are a number of well-established processes for producing deep, smooth, and highly vertical-etched structures in SOI. One of the key advantages of planar grating devices compared to freespace bulk gratings is that they are lithographically produced and any grating shape is readily achievable. Techniques for etching silicon have benefited from recent advancements in the fabrication of microelectromechanical systems. Planar reflective gratings in SOI have the potential for monolithic integration with large-scale electronic-integrated circuits.

An SOI-based planar grating uses a variety of input and output structures, as shown in Figure 1. In a rib-waveguide SOI structure, the fundamental mode of the waveguide is asymmetrically positioned below the center of the slab core. In order to improve the waveguide-to-slab coupling, the input waveguide is usually tapered at the entrance to the slab region. In the slab, the light remains confined in the vertical direction but expands horizontally until it encounters a series of reflectors. The grating not only diffracts but also refocuses the light into the output waveguides, as shown in Figure 1.

The lithographic approach to making planar gratings carries little design restrictions on the shape of the launching mode or the shapes of the diffracting elements. Such flexibility in planar grating design has recently resulted in the successful demonstration of planar gratings with facet widths varying from 4 to $1000 \mu \mathrm{m}[4,7]$. Diffractive elements in SOI can be created by either a single dielectric-to-metal interface [5], a reflective dielectric stack [8], or using total-internal reflection facets [6].

The first planar reflective gratings were realized using the Rowland configuration [9]. Research into novel grating

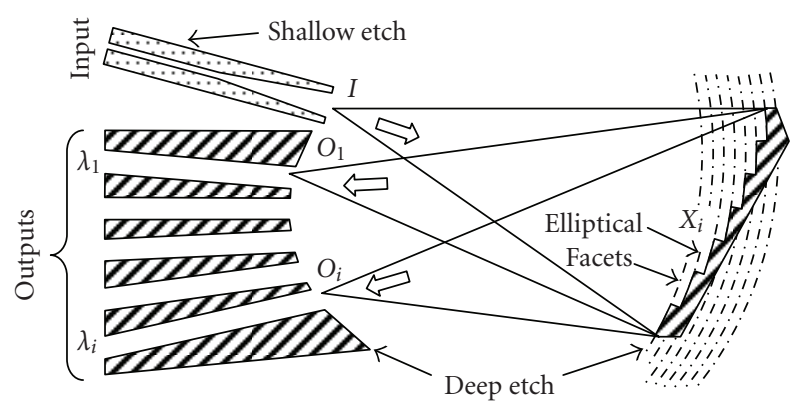

FIGURE 1: Architecture of the planar reflective grating in a siliconon-insulator optical interconnect. A shallow etch $(0.5 \mu \mathrm{m})$ defines the input structure, whereas a deep etch $(1.7 \mu \mathrm{m})$ defines the output waveguides and grating facets.

designs has since yielded a methodology for eliminating the aberration effects caused by grating facets [10]. Subsequently, a mechanism of compensating for the dispersion of the refractive index of glass has been discovered, resulting in the successful demonstration of interleavers [7].

A comparative analysis of both planar reflective gratings and arrayed waveguide gratings recently performed by Cheben found that the traditional scalar approach to the simulation of planar gratings is unable to reconcile simulated and measured data [11]. Numerical difficulties posed by the large number of diffraction orders, deep triangular grooves with high blaze angles, and small wavelength-tograting pitch ratios have made the calculation of diffraction efficiency one of the most intractable problems of electromagnetic theory.

Until a decade ago, simple relationships between fundamental grating parameters, such as diffraction order, diffraction angle, resolving power, Rowland circle radius, grating pitch, were deemed sufficient to model planar reflective gratings. The linear dispersion strength of a planar grating can be readily computed through the angle of incidence, $\alpha$, and reflected angle, $\alpha^{\prime}[12]$ :

$$
L \frac{\partial \alpha^{\prime}}{\partial \lambda}=L \frac{\sin \alpha+\sin \alpha^{\prime}}{\lambda \cos \alpha^{\prime}}\left[1-\frac{\lambda}{n} \frac{\partial n}{\partial \lambda}\right]
$$

where $L$ is the length of the slab region. Theoretically, it is possible to use infinite permutations of diffraction angles and slab lengths to produce gratings with almost identical characteristics. However, in practice, researchers have realized that theoretically equivalent gratings can exhibit vastly different performance characteristics.

Vector diffraction theories that replaced the traditional scalar approach revealed that the shape and metallization of the grating facets affect both the grating efficiency and polarization sensitivity. Delâge and Dossou [13] solved Maxwell's equations in proximity to the grating facets using a finite-element method and matched their numerical solution near the grating surface to a Rayleigh expansion. Their work showed that metallization had a profound impact on the absolute and relative grating efficiencies of both TE and TM polarized light. 
The design of planar gratings in SOI requires careful studies on the effects of silicon core thickness and grating verticality on the polarization dependence and optical crosstalk. Particular consideration should be given to the selection of diffraction angles for the planar grating in SOI.

\section{POLARIZATION DEPENDENCE OF PLANAR GRATINGS}

The polarization dependence of the wavelength (PDW) has different origins in AWGs and planar reflective gratings. PDW in an SOI-based AWG is predominantly caused by waveguide birefringence in the phase array region. Contributing factors to the waveguide birefringence include waveguide cross-section variations and stress. Both of these factors can be used to eliminate modal birefringence in SOIridge waveguides [14]. Consequently, the selection of proper waveguide dimensions and cladding layers is essential for improving the PDW performance of AWGs.

Contrary to AWGs, the PDW of SOI planar waveguide gratings depends only on birefringence of the slab and not the waveguides. The geometry of waveguides can be selected independently, thus providing additional design flexibility in optimizing other planar lightwave circuit subcomponents, such as fiber-to-waveguide mode converters and bend waveguide regions.

The modes of an asymmetric slab waveguide can be found using analytical expressions that assume that the waveguide is infinite in the transverse direction [15]. The three layers of the infinite asymmetric slab waveguide are a cladding layer of air $\left(n_{0}=1\right)$, a core layer of silicon $\left(n_{1}=3.48\right)$, and a substrate layer of $\mathrm{SiO}_{2}\left(n_{2}=1.445\right)$. The thickness of the cladding and substrate layers are assumed to be infinite in these calculations, a good approximation due to the strong modal confinement in the high-index silicon core. The equations for the TM mode are found using the duality relationships between TE and TM electric and magnetic fields. The computation was performed for a wavelength of $1.55 \mu \mathrm{m}$.

The results of the effective index computations are shown in Figure 2 (left axis). The effective index of the fundamental slab mode in a silicon core has been found to depend strongly on the core thickness, due to the high index contrast of SOI. The birefringence of the slab waveguide becomes greater as the core thickness decreases, with only the TE mode propagating at core thicknesses of $<0.12 \mu \mathrm{m}$. In silica, researchers have found a way of eliminating the slab birefringence through a use of a compensator region [16]. In SOI, the amount of birefringence is orders of magnitude larger.

Polarization compensation in SOI is possible for a range of core thicknesses using, for example, the approach described in [16]. The difficulty associated with this approach is that a large mode profile mismatch between the slab and compensator regions can excite undesirable higher modes. This problem has been solved by Cheben et al. using silicon-oxide-silicon compensators that can provide

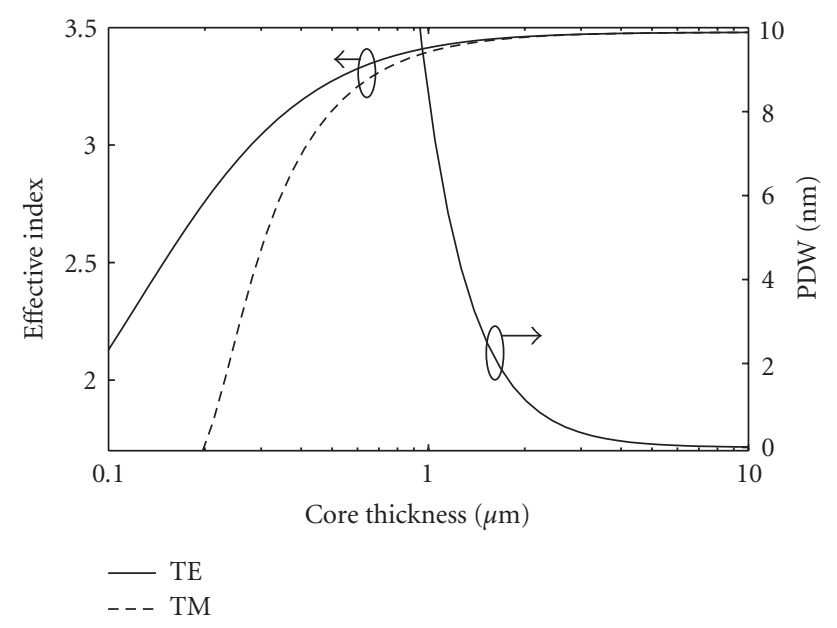

Figure 2: (i) Dependence of the effective refractive index of the fundamental mode on the core thickness of the slab for TE- and TM-polarized lights (left axis); and (ii) polarization dependence of the wavelength as a function of the slab thickness (right axis).

sufficiently strong birefringence correction with minimal field profile mismatch $[17,18]$.

The present manuscript is limited only to the consideration of planar gratings containing no polarization compensators. The design and architecture of compensators is a subject of our present research in this field. A strong slab birefringence has a profound effect on the optical properties of silicon interconnects based on planar reflective gratings. In Figure 2 (right axis), we have computed the amount of PDW that is caused by slab birefringence. The data clearly shows that, in devices with a silicon core thickness of $<1 \mu \mathrm{m}$ (PDW $=\lambda \cdot \Delta n / n>10 \mathrm{~nm})$, it is not possible to achieve polarization-independent performance for most practical applications. In a different approach, it has been suggested that the polarization diversity scheme can potentially be applied to overcome the polarization dependence of reflective interconnects [19]. This, however, would likely lead to doubling the number of optical and electronic subcomponents and would also require integrating polarization splitters and combiners. The complexity of the polarization diversity scheme will likely outweigh the benefits of silicon photonic integration for the majority of applications that require polarization insensitivity.

In addition to PDW, SOI-based devices also experience polarization-dependent loss (PDL). Nanometer-scale sidewall roughness is the prime cause of polarization-dependent waveguide loss in silicon waveguides [20]. In AWGs, PDL primarily arises from different waveguide propagation losses associated with the two polarizations in the phase array region. Planar reflective gratings, in contrast, require no phase-array waveguides, but rather a slab region that can be made polarization-insensitive. However, PDL does arise due to the presence of nonreflecting sidewalls between adjacent grating facets. The approach to reduce this source of PDL will be discussed later in this manuscript. 


\section{OPTICAL CROSSTALK}

Optical crosstalk also has different origins for AWGs and planar reflective gratings. In AWGs, the theoretical crosstalk limit depends on the number of waveguides (the Gaussian field truncation) and waveguide phase errors. The Gaussian field truncation can be overcome by merely increasing the number of waveguides in the phase array region. However, controlling the phase noise in SOI waveguides is very challenging. Even nanometer-scale variations in the waveguide geometry leads to severe phase distortions. Phase error noise in SOI typically results in optical crosstalk values unsuitable for most applications [3]. This limitation underscores the difficulty of implementing the AWG architecture in SOI.

Planar reflective gratings offer the unique possibility of overcoming the crosstalk limitations of the SOI platform. The optical crosstalk of planar reflective gratings is not degraded by waveguide geometry phase errors since diffraction occurs in the slab region. The slab region typically has a high refractive index and thickness uniformity. The two main contributors to optical crosstalk in planar gratings are grating anomalies and facet verticality. A numerical model which simulates the spectral characteristics of the demultiplexer including grating anomalies due to the pixilation effect, random stage movement error, and gain error has already been established and discussed in the literature [21]. In this study, we primarily examine the effects of grating verticality on the cumulative optical crosstalk.

In a multichannel demultiplexer, the cumulative optical crosstalk arising from a grating facet tilted by $\theta$ degrees from the vertical can be computed by considering the phase distortion of the mode reflected from the facet. For the TE mode (and similarly for the TM mode) the mode reflected from a tilted interface, $\mathbf{E}_{\text {tilted }}$, is given by the input fundamental slab mode, $\mathbf{E}_{\text {slab }}$, multiplied by a phase-shifting factor

$$
\mathbf{E}_{\text {tilted }}=\mathbf{E}_{\text {slab }} e^{i 2 \beta y \tan \theta},
$$

where $y$ is the coordinate in the growth direction and $\beta$ is the slab mode propagation constant. Crosstalk originates from the fact that the reflected mode does not perfectly couple with the fundamental slab mode, and the uncoupled portion of the light can contribute to the crosstalk. Assuming that all fields are normalized, the amount of cumulative crosstalk can be estimated by calculating the coupling loss between the reflected mode and the fundamental slab mode (wavelength dependence is implicit):

$$
\begin{aligned}
& \eta_{\mathrm{TE}}=1-\left|\frac{1}{2} \int_{-\infty}^{\infty} E_{y, \text { slab }} H_{x, \text { tilted }}^{*} d x\right|^{2}, \\
& \eta_{\mathrm{TM}}=1-\left|\frac{1}{2} \int_{-\infty}^{\infty} E_{x, \text { slab }} H_{y, \text { tilted }}^{*} d x\right|^{2} .
\end{aligned}
$$

This gives the worst case crosstalk estimate, as some of the reflected mode may couple to radiation modes and not contribute to the crosstalk. In multichannel planar grating demultiplexers, the spectral response from each channel, including side-lobes and other crosstalk limiting features,

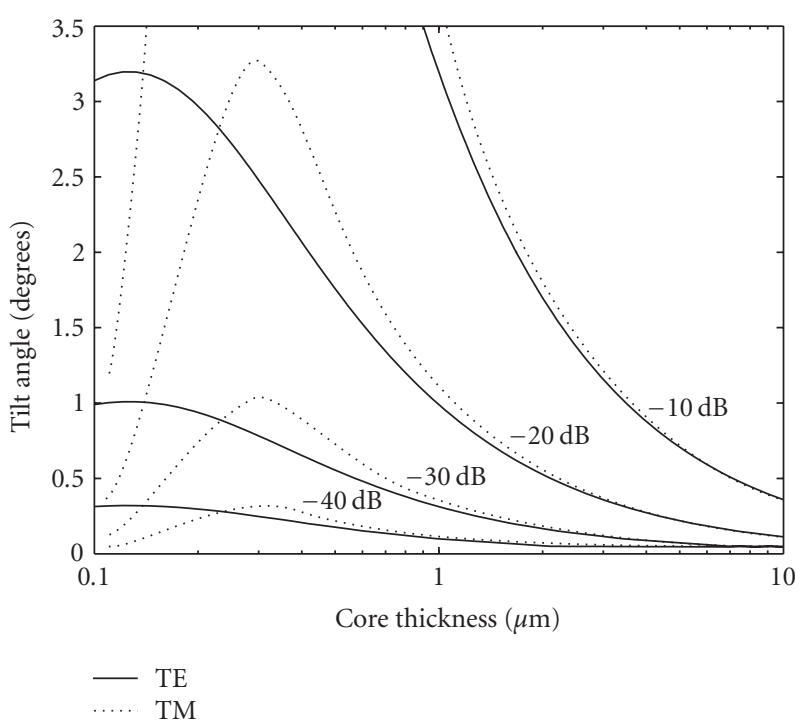

Figure 3: Cumulative optical crosstalk as a function of silicon core thickness and facet tilt angle (relative to vertical) for different isolation requirements. In order to achieve $-30 \mathrm{~dB}$ isolation in 1.7$\mu$ m-thick silicon slab, the verticality of facets should be controlled to within \pm 0.4 degree.

is very similar if channels are spectrally shifted by the amount of channel spacing and overlaid [12]. Higher-order slab modes have different propagation constants and will generally diffract at different angles, focusing at spatially different locations along the Rowland circle, resulting in "ghost peaks" appearing in the other channels. Effectively, each channel will experience crosstalk from a different spectral window coming from every other channel. Thus, the cumulative optical crosstalk can be estimated by either adding together crosstalks in different spectral windows from all the channels, or alternatively performing a spectral integral of crosstalk coming from a single channel, as given by the above formulas.

The results of our simulations are shown in Figure 3. Each curve shows the maximum vertical tilt angle of the grating permissible for a given silicon core thickness so as to maintain a particular amount of cumulative crosstalk, from $-10 \mathrm{~dB}$ to $-40 \mathrm{~dB}$. From Figure 3, silicon cores with a thickness of $\sim 0.3 \mu \mathrm{m}$ are capable of withstanding the largest grating facet tilt with the smallest amount of crosstalk. For thicker silicon cores, crosstalk values become unacceptable even for relatively vertical grating facets (e.g., crosstalk $\eta_{\mathrm{TE}} \approx$ $\eta_{\mathrm{TM}}=-15 \mathrm{~dB}$ for core thickness of $5 \mu \mathrm{m}$ and a tilt angle of $\left.\theta=0.5^{\circ}\right)$.

Figure 3 also shows the polarization dependence of the cumulative crosstalk. For thinner cores $(<0.2 \mu \mathrm{m})$, TMpolarized light is more sensitive to the facet tilt angle, while for larger core thicknesses TE-polarized light is more sensitive. For reflective gratings with thick cores $(>10 \mu \mathrm{m})$, the TE- and TM-polarized modes are both equally highly sensitive to the tilt angle. These results can be explained by examining the confinement of the optical mode. As the optical power in the mode becomes more diffuse in the 
vertical direction, the edges of the modal field experience a greater phase shift according to (2), and the mode will become more sensitive to grating verticality. The peaks in Figure 3 occur at core thicknesses where the TE or TM mode is the most vertically confined.

Figure 4 shows the confinement in the silicon core of the TE- and TM-polarized mode. For thinner cores, where the modes are only weakly guided, the TM mode is much less confined than the TE mode, resulting in a greater sensitivity to the tilt angle. As the core thickness increases, the TMpolarized light becomes more confined than TE-polarized light, and hence less sensitive to the tilt angle. At core thicknesses above $1 \mu \mathrm{m}$, both TE- and TM-polarized modes are almost entirely confined in the core, and the width of the mode field in the vertical direction increases as the core thickness increases. This results in a high sensitivity to the tilt angle at large values of the core thickness.

It is advantageous to use the thinnest possible core that provides acceptable PDW values (see Figure 2). Thus, the selection of the core thickness has to be done in conjunction with a specific application. Irrespective of the core thickness, the ability to etch nearly vertical grating facets is essential in minimizing the insertion loss and reducing the optical crosstalk of planar reflective gratings.

\section{CWDM DEMULTIPLEXER DESIGN}

We have applied our findings to design an SOI-based CWDM demultiplexer suited for use in integrated receiver applications. The CWDM grid is made up of 18 wavelengths defined within a range of $1270 \mathrm{~nm}$ to $1610 \mathrm{~nm}$, spaced by $20 \mathrm{~nm}$ [22]. CWDM can support various topologies of interconnectshubbed rings, point-to-point, and passive optical networks with transmission distances of up to $50 \mathrm{~km}$. The CWDM receivers often require larger bandwidths that can capture all the specified bitrates and protocols. We assume that our CWDM demultiplexer is used in conjunction with wavelength agnostic PINs or avalanche photodiode detectors (APDs) that operate over the entire ITU CWDM band.

On the transmitter side, polarization control is not necessary since laser diodes are strongly polarized. However, on the receiver side, the incoming signal polarization is not known a priori, consequently, receiver demultiplexers should be sufficiently polarization insensitive. From Figure 2, it is apparent that to have sufficiently low polarization dependence $(\mathrm{PDW}<2 \mathrm{~nm}$ ) in a planar reflective grating containing no polarization compensators, the silicon core thickness should be no less than $1.7 \mu \mathrm{m}$. On the other hand, for the best crosstalk performance, Figure 3 suggests that the core thickness is as small as possible. Therefore, to construct the CWDM receiver, we selected the nominal core thickness of $1.7 \mu \mathrm{m}$.

The rib waveguide geometry was chosen to be single mode according to the modified criterion of Soref et al. [23]. Single-mode operation was confirmed with a fullvectorial finite-element method. The nominal ridge width was chosen to be $1.5 \mu \mathrm{m}$ with a ridge depth of $0.5 \mu \mathrm{m}$. The TM mode profile for this structure, generated using finiteelement analysis, is shown in Figure 5.

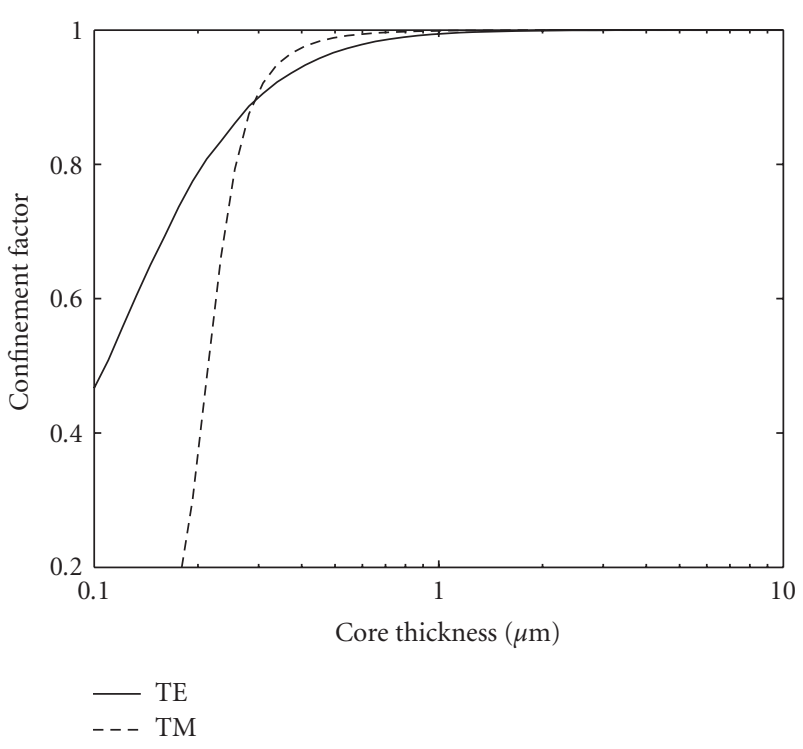

FIgURE 4: Confinement factor in the core layer of TE- and TMpolarized lights versus thickness of the core layer.

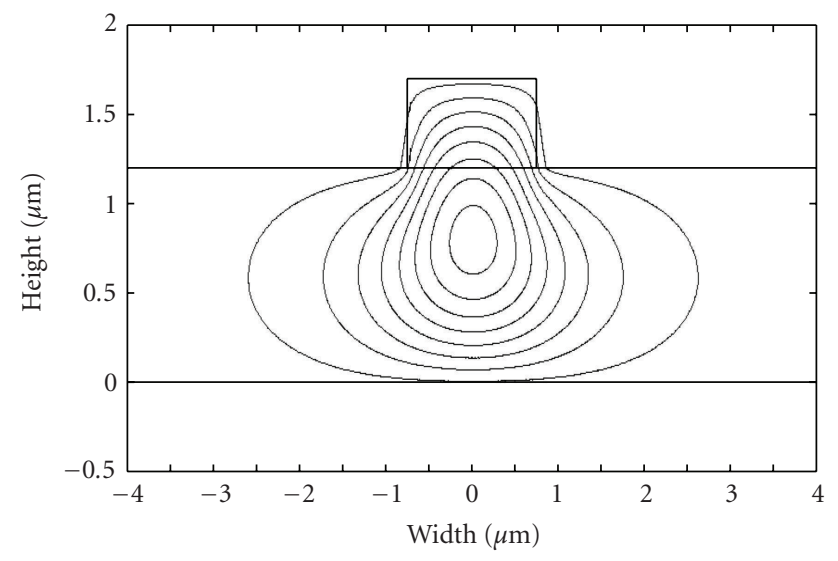

FIGURE 5: The TM mode profile of the input rib waveguide of the CWDM demultiplexer. Birefringence of the waveguide does not contribute to the polarization dependence of a planar reflective grating.

The high-contrast silicon slab considered here supports a large number of higher-order modes. Consequently, special consideration should be given to the design of the input taper to prevent higher-order mode excitation. If higher-order modes are unintentionally excited, it will inevitably lead to the presence of ghost peaks in the output guides even if the grating facets are perfectly vertical. To overcome the higherorder mode excitation, the input waveguide incorporates a linear taper that adiabatically increases the rib width from $1.5 \mu \mathrm{m}$ to $4 \mu \mathrm{m}$ over a length of $2000 \mu \mathrm{m}$. The length of the slab region was chosen to be $6200 \mu \mathrm{m}$. The slab length is selected to achieve sufficient linear dispersion (as given by (1)) to accommodate the width and separation between the output waveguides. 
The selection of the diffraction grating order is dictated by the requirements imposed by the free spectral range (FSR) of the device. In general, the overlap between different orders is determined by

$$
(N+1) \lambda_{1}=N \lambda_{2}
$$

In order to accommodate all 18 channels spaced at $20 \mathrm{~nm}$, as required for a CWDM receiver, $\Delta \lambda=\lambda_{2}-\lambda_{1}$ should be more than $400 \mathrm{~nm}$. Such a wide-free spectral range demands the diffraction order to be $N=1$, yielding a free spectral range of over $600 \mathrm{~nm}$. While low-diffraction order devices are easily achievable using the planar reflective grating technology, realizing such wide-free spectral range device using the AWG architecture would require an impractically large phase array region that would have severe crosstalk limitations in SOI.

The Rowland circle configuration was chosen as a zeroorder approximation to the position of refractive elements and the plane of the input and output waveguides. The firstorder correction was obtained through the use of standard double-astigmatic point improvement to the position of the grating facets [11]. In our case, we chose $1270 \mathrm{~nm}$ and $1610 \mathrm{~nm}$ as astigmatic wavelengths to compensate for the reduced diffraction efficiency in the end channels. The energy of light diffracted by the grating was optimized by selecting a blaze wavelength of $1440 \mathrm{~nm}$.

Careful consideration should be given to the selection of diffraction angles for the planar grating in SOI. Traditionally, planar reflective gratings in SOI have been selected to have incident and reflected angles of around 40 degrees $[4,6]$. However, building a wide-free spectral range CWDM demultiplexer at such large diffracting angles would lead to suboptimally shaped grating facets that are susceptible to corner rounding and polarization dependence. While it is possible to remove reflective material from the nonreflective facets to improve the grating efficiency, this would require additional postprocessing steps such as selective metal etch removal.

The reduction in the planar grating diffraction efficiency due to corner rounding is approximately equal to the ratio between the size of the round corner and the size of the reflective portion of the facet. It is advantageous to have the size of the reflective facet much larger compared to the corner rounding. Furthermore, the ratio of the reflective to nonreflective facet has been shown to be the key for overcoming PDL in planar reflective gratings [24]. Consequently, the diffraction angle should be low enough to produce sufficiently wide reflecting facets for ease of manufacturing, while keeping the size of the nonreflecting facets small enough to eliminate corner rounding and polarization effects.

Our analysis of the grating parameters yielded a value of 3.5 degrees for the angle of diffraction. At this diffraction angle, an average size of the reflective facet is $3 \mu \mathrm{m}$ while the nonreflecting sidewall is only $0.2 \mu \mathrm{m}$. This results in the aspect ratio of the reflecting to nonreflecting facet of 15 : 1 , putting a limit of $-0.3 \mathrm{~dB}$ on the maximum diffraction efficiency loss due to corner rounding. The total number of facets was chosen to be 600 . This ensured that well over

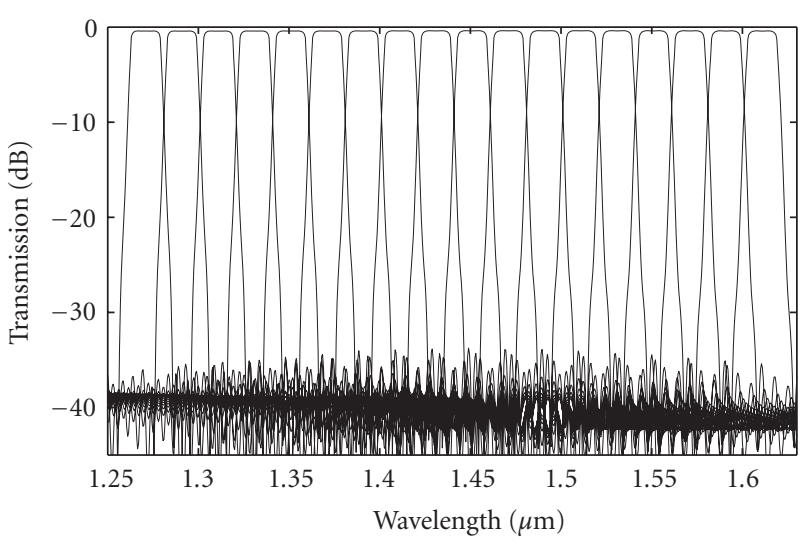

Figure 6: Transmission spectra of an 18-channel CWDM demultiplexer for TM polarization. No decrease in the diffraction efficiency was observed for the end channels. Optical crosstalk of better than $-35 \mathrm{~dB}$ was achieved.

99\% of the light from the input waveguide is captured by the grating. Each grating facet was chosen to be an elliptical shape to further reduce aberration effects. Details of aberration reduction are described in [10].

To achieve a box-like channel profile for the CWDM demultiplexer, the output waveguides are formed using a $1.7-\mu \mathrm{m}$-deep etch with a waveguide width of $8 \mu \mathrm{m}$ and a separation between waveguides of approximately $4 \mu \mathrm{m}$ (see Figure 1). This output waveguide structure can support a large number of modes. Since the proposed device is specifically designed for use in receiver applications, we assume that the active area of the photodetector is sufficiently large to absorb all of the light in the multimode guide.

To simulate the grating performance, we used a fullvectorial finite-element method to compute the modes of input and output guides. An asymptotic approximation for Hankel functions of large argument was then used to obtain the intensity on the grating facets. This approximation was used once again to obtain the reflected field $\Theta(\lambda)$ on the plane of the output waveguide structures. The demultiplexer transmission intensity at a specific wavelength was obtained by a convolution of the diffracted light with the intensity profile of each individual mode $\varphi_{i}(\lambda)$ in each output multimode output waveguide:

$$
T(\lambda)=\sum_{i}\left\langle\Theta(\lambda) \mid \phi_{i}(\lambda)\right\rangle .
$$

The results of our simulation are shown in Figure 6 for TM polarization. It is worth pointing out that the diffraction efficiency at the end channels is as strong as the efficiency of the central channels. This was possible by our selection of the blaze wavelength of the planar grating to be in the middle of the free spectral range, and the use of the end wavelengths for astigmatic points. Crosstalk values of better than $-35 \mathrm{~dB}$ have been computed for all 18 channels. The 0.5 -dB spectral width of an individual channel was estimated to be $16 \mathrm{~nm}$. Considering that this device has a projected PDW of $2 \mathrm{~nm}$ due to the slab birefringence, centering the wavelength between the two extreme polarizations can potentially yield 
$15 \mathrm{~nm}$ of useful bandwidth for each individual channel. Such wide spectral passbands can be useful for transmitting large-bandwidth data while still allowing for the temperature drift of the demultiplexer and the transmitter laser diodes. This work confirms that planar reflective gratings in SOI can be used to achieve both wide-free spectral range and polarization-insensitive performance.

Multiplexing and demultiplexing light is only one of many possible applications of planar reflective gratings in silicon interconnects. Planar reflective gratings can also be used in add-drop filters, repeaters, dispersion compensators, and gain equalizers. Because of their reflective nature, planar gratings can be very effective in stabilizing laser sources through wavelength locking. Additional applications of planar gratings will likely be found in optical clocking through the use of time domain to frequency domain interferometry in silicon interconnects.

\section{SUMMARY}

In summary, we have investigated the optical properties of planar reflective gratings for building optical interconnects in silicon on insulator for various silicon core thicknesses. We have shown that the dispersion of the slab can be controlled through the core thickness selection to produce polarizationinsensitive gratings. The verticality of the grating facets has been found to be the main contributing factor to the cumulative crosstalk in thick silicon cores. Our findings were applied towards the design and simulation of an 18-channel coarse wavelength division demultiplexer that has both a widefree spectral range and polarization-insensitive performance. These results demonstrate that planar reflective gratings can become an essential element in building many silicon photonics applications.

\section{REFERENCES}

[1] A. W. Fang, H. Park, O. Cohen, R. Jones, M. J. Paniccia, and J. E. Bowers, "Electrically pumped hybrid AlGaInAs-silicon evanescent laser," Optics Express, vol. 14, no. 20, pp. 92039210, 2006

[2] H. Park, A. W. Fang, R. Jones, et al., "A hybrid silicon evanescent photodetector," in Proceedings of the 65th Annual Device Research Conference (DRC '07), pp. 185-186, South Bend, Ind, USA, June 2007.

[3] W. Bogaerts, P. Dumon, D. Van Thourhout, et al., "Compact wavelength-selective functions in silicon-on-insulator photonic wires," IEEE Journal on Selected Topics in Quantum Electronics, vol. 12, no. 6, pp. 1394-1401, 2006.

[4] J. Brouckaert, W. Bogaerts, P. Dumon, D. Van Thourhout, and R. Baets, "Planar concave grating demultiplexer fabricated on a nanophotonic silicon-on-insulator platform," Journal of Lightwave Technology, vol. 25, no. 5, pp. 1269-1275, 2007.

[5] S. Bidnyk, D. Feng, A. Balakrishnan, et al., "Silicon-oninsulator-based planar circuit for passive optical network applications," IEEE Photonics Technology Letters, vol. 18, no. 22, pp. 2392-2394, 2006.

[6] W. Wang, Y. Tang, Y. Wang, et al., "Etched-diffractiongrating-based planar waveguide demultiplexer on silicon-oninsulator," Optical and Quantum Electronics, vol. 36, no. 6, pp. 559-566, 2004.
[7] S. Bidnyk, A. Balakrishnan, A. Delâge, et al., "Novel architecture for design of planar lightwave interleavers," Journal of Lightwave Technology, vol. 23, no. 3, pp. 1435-1440, 2005.

[8] J. Brouckaert, W. Bogaerts, P. Dumon, et al., "Planar concave grating demultiplexer with distributed bragg reflection facets," in Proceedings of the 4th IEEE International Conference on Group IV Photonics, pp. 1-3, Tokyo, Japan, September 2007.

[9] H. W. Yen, H. R. Friedrich, R. J. Morrison, and G. L. Tangonan, "Planar Rowland spectrometer for fiber-optic wavelength demultiplexing," Optics Letters, vol. 6, no. 12, pp. 639-641, 1981.

[10] S. Bidnyk, A. Balakrishnan, A. Delâge, et al., "Planar comb filters based on aberration-free elliptical grating facets," Journal of Lightwave Technology, vol. 23, no. 3, pp. 1239-1243, 2005.

[11] P. Cheben, "Wavelength dispersive planar waveguide devices: echelle gratings and arrayed waveguide gratings," in Optical Waveguides: From Theory to Applied Technologies, M. L. Calvo and V. Laksminarayanan, Eds., chapter 5, CRC Press, London, UK, 2007.

[12] S. Bidnyk, A. Balakrishnan, M. Pearson, M. Gao, and T. Hall, "Configurable coarse wavelength division demultiplexers based on planar reflective gratings," Optics Communications, vol. 262, no. 1, pp. 82-86, 2006.

[13] A. Delâge and K. Dossou, "Polarisation dependent loss calculation in echelle gratings using finite element method and Rayleigh expansion," Optical and Quantum Electronics, vol. 36, no. 1-3, pp. 223-238, 2004.

[14] D.-X. Xu, P. Cheben, D. Dalacu, et al., "Eliminating the birefringence in silicon-on-insulator ridge waveguides by use of cladding stress," Optics Letters, vol. 29, no. 20, pp. 23842386, 2004.

[15] S. L. Chuang, Physics of Optoelectronic Devices, WileyInterscience, New York, NY, USA, 1995.

[16] J. J. He, E. S. Koteles, L. Erickson, B. Lamontagne, and A. Delâge, "Optical grating-based device having a slab waveguide polarization compensating region," US patent 5937113, 1999.

[17] P. Cheben, D.-X. Xu, S. Janz, A. Delâge, and D. Dalacu, "Birefringence compensation in silicon-on-insulator planar waveguide demultiplexers using a buried oxide layer," in Photonics Packaging and Integration III, vol. 4997 of Proceedings of SPIE, pp. 181-189, San Jose, Calif, USA, January 2003.

[18] P. Cheben, D.-X. Xu, S. Janz, and A. Delâge, "Scaling down photonic waveguide devices on the SOI platform," in VLSI Circuits and Systems, vol. 5117 of Proceedings of SPIE, pp. 147156, Maspalomas, Spain, May 2003.

[19] W. Bogaerts, D. Taillaert, P. Dumon, E. Pluk, D. Van Thourhout, and R. Baets, "A compact polarization-independent wavelength duplexer using a polarization-diversity SOI photonic wire circuit," in Proceedings of the Optical Fiber Communication and the National Fiber Optic Engineers Conference (OFC/NFOEC '07), pp. 1-3, Anaheim, Calif, USA, March 2007, paper OTuM2.

[20] D. K. Sparacin, S. J. Spector, and L. C. Kimerling, "Silicon waveguide sidewall smoothing by wet chemical oxidation," Journal of Lightwave Technology, vol. 23, no. 8, pp. 2455-2461, 2005.

[21] J.-J. He, B. Lamontagne, A. Delâge, L. Erickson, M. Davies, and E. S. Koteles, "Monolithic integrated wavelength demultiplexer based on a waveguide Rowland circle grating in InGaAsP/InP," Journal of Lightwave Technology, vol. 16, no. 4, pp. 631-637, 1998.

[22] International Telecommunication Union, ITU-T Recommendation G.694.2, 2002. 
[23] R. A. Soref, J. Schmidtchen, and K. Petermann, "Large singlemode RIB waveguides in GeSi-Si and $\mathrm{Si}-$ on-SiO ${ }_{2}$," IEEE Journal of Quantum Electronics, vol. 27, no. 8, pp. 1971-1974, 1991.

[24] S. Bidnyk, A. Balakrishnan, and M. Pearson, "Planar waveguide reflective diffraction grating," US patent 7151635, 2006. 

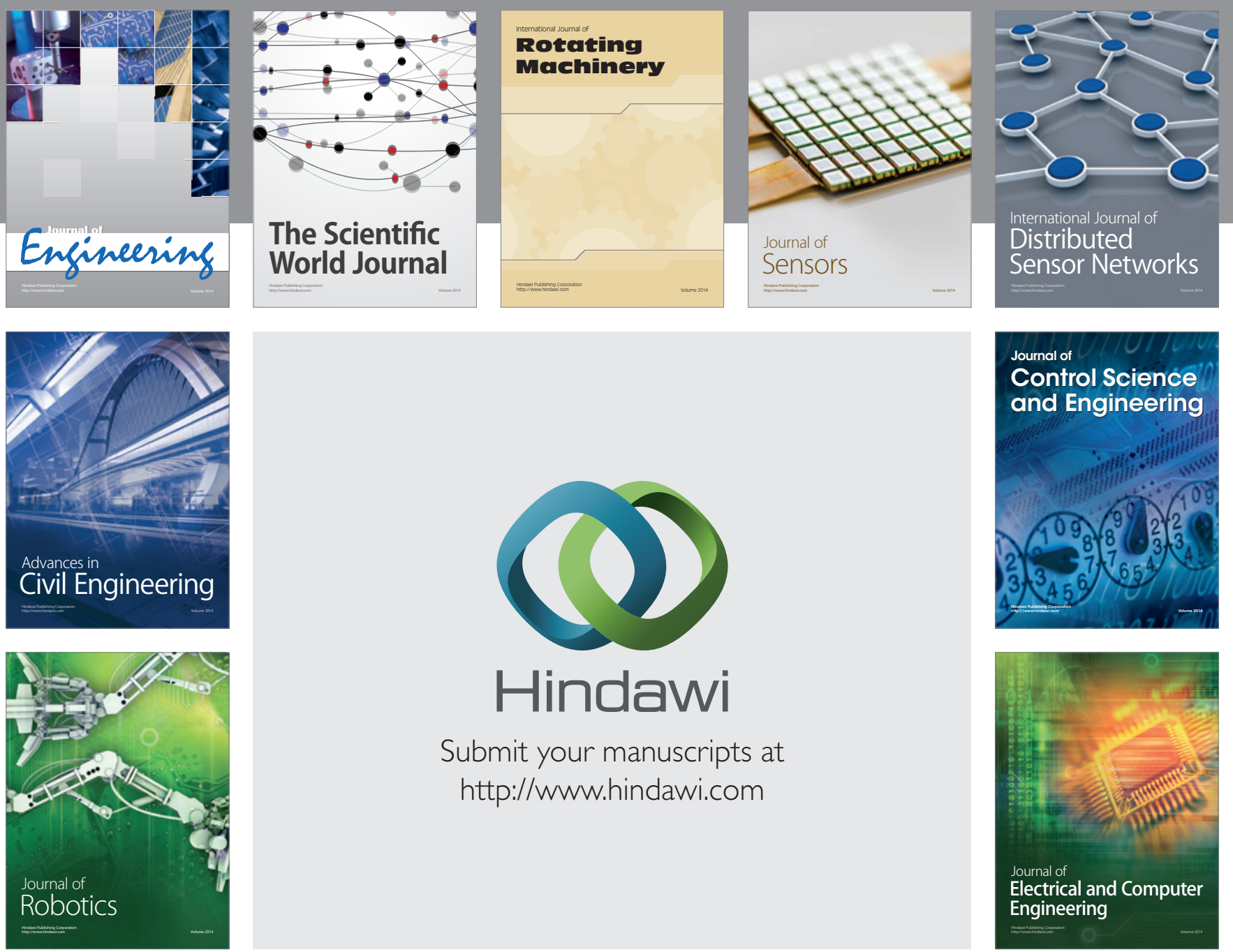

Submit your manuscripts at

http://www.hindawi.com
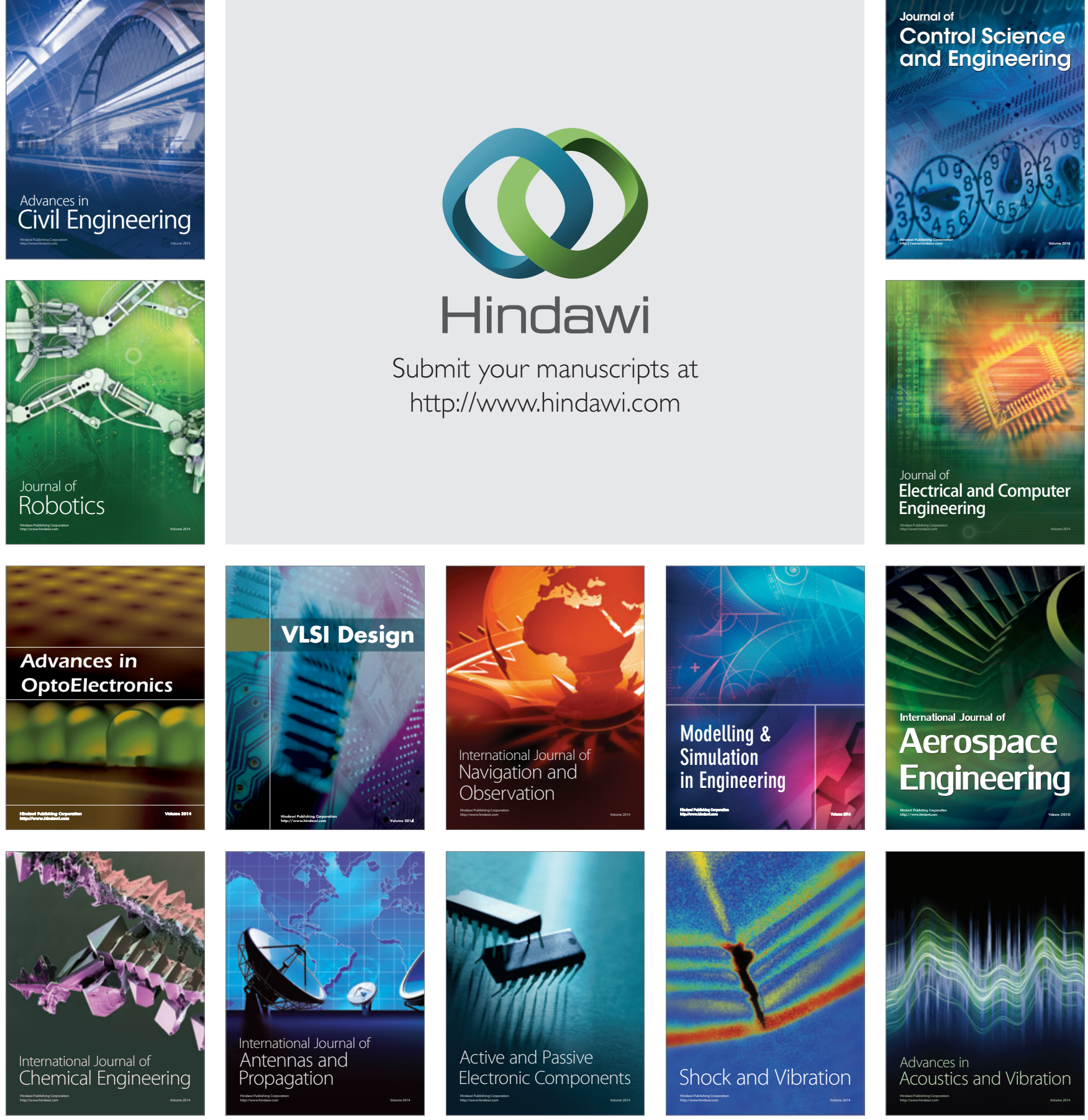